\title{
Artifact for Enhancing Genetic Improvement of Software with Regression Test Selection
}

\author{
Giovani Guizzo*, Justyna Petke*, Federica Sarro* and Mark Harman*† \\ ${ }^{*}$ Department of Computer Science, University College London (UCL), London, United Kingdom \\ ${ }^{\dagger}$ Facebook, London, United Kingdom \\ \{g.guizzo, j.petke, f.sarro, mark.harman\}@ucl.ac.uk
}

\section{INTRODUCTION}

We present in this document the basic information needed to download, unpack, and then interpret the instructions we provide as requested in the ICSE 2021 Artifact Submission Guidelines. The artifact contains all the subject programs, scripts, tools, results, and a series of guidelines on how to use them.

We aim at obtaining the badges of Available and Reusable. In order to do so, we have added all the components needed for the full execution of the experiments and analyses as we originally did during the writing of our paper, making it readily available. Furthermore, we have included instructions to the INSTALL file on how to add new programs to the experiments and analyses, making it reusable for the next researchers that intend to replicate or extend our experiments.

\section{DOI AND WHERE TO DOWNLOAD}

We uploaded the experimental package to UCL's Figshare. We have reserved the following DOI: 10.5522/04/12890792. The link to download the package is https://doi.org/10.5522/ 04/12890792.

We also provide a Docker image at https://hub.docker.com/ repository/docker/giovaniguizzo/icse21-p66. All the instructions on how to use it are contained in the INSTALL file.

\section{UNPACKING ThE PACKAGE}

The package is compressed into a . zip file of roughly 360MB. After downloading, one can unpack it to any convenient directory. It will create a directory named "ICSEExperimental-Package" by default with roughly $1 \mathrm{~GB}$ of content.

\section{Getting Started}

Everything needed to run the experiments and data analysis can be found in the README.md, REQUIREMENTS.md, and INSTALL. md files. We also provide a few additional files to describe which badges we are aiming for (STATUS.md), the authors' details and contacts (AUTHORS .md), and our license (LICENSE.md). Our accepted paper is also included in the package (PAPER.pdf).

To ease the reading, all .md files were also compiled into .html and .pdf files. The content is the same in all three versions, thus the reader can choose to read the type of file according to their likings.
We advise the reader to start by reading the README instructions, which contains basic information about the content of the package. This file describes all the other files, including the other instruction files.

REQUIREMENTS is the second file we advise one to read, which contains software and hardware requirements needed to run the experiments and data analysis.

Finally, the INSTALL file contains all the instructions to prepare the Docker image or to install the required dependencies locally, to run the experiments with output examples, to add new programs to the experiments and data analysis, and to perform the data analysis.

Since the examples of usage are quite long, we refer the reader to those instruction files, as opposed to adding their content to this manuscript.

\section{Contact}

In case of any question, the reader can contact the first author, Dr. Giovani Guizzo, via e-mail at g.guizzo@ucl.ac.uk. 